\title{
Understanding NMR relaxometry of partially water-saturated rocks
}

\author{
O. Mohnke, R. Jorand, C. Nordlund, and N. Klitzsch \\ Institute for Applied Geophysics and Geothermal Energy (GGE), E.ON Energy Research Center (E.ON ERC), \\ RWTH Aachen University, Mathieustrasse 10, 52074 Aachen, Germany
}

Correspondence to: O. Mohnke (omohnke@eonerc.rwth-aachen.de)

Received: 16 September 2014 - Published in Hydrol. Earth Syst. Sci. Discuss.: 17 November 2014

Revised: 23 April 2015 - Accepted: 10 May 2015 - Published: 16 June 2015

\begin{abstract}
Nuclear magnetic resonance (NMR) relaxometry measurements are commonly used to characterize the storage and transport properties of water-saturated rocks. Estimations of these properties are based on the direct link of the initial NMR signal amplitude to porosity (water content) and of the NMR relaxation time to pore size. Herein, pore shapes are usually assumed to be spherical or cylindrical. However, the NMR response at partial water saturation for natural sediments and rocks may differ strongly from the responses calculated for spherical or cylindrical pores, because these pore shapes do not account for water menisci remaining in the corners of desaturated angular pores. Therefore, we consider a bundle of pores with triangular cross sections. We introduce analytical solutions of the NMR equations at partial saturation of these pores, which account for water menisci of desaturated pores. After developing equations that describe the water distribution inside the pores, we calculate the NMR response at partial saturation for imbibition and drainage based on the deduced water distributions.

For this pore model, the NMR amplitudes and NMR relaxation times at partial water saturation strongly depend on pore shape, i.e., arising from the capillary pressure and pore shape-dependent water distribution in desaturated pores with triangular cross sections. Even so, the NMR relaxation time at full saturation only depends on the surface-to-volume ratio of the pore. Moreover, we show the qualitative agreement of the saturation-dependent relaxation-time distributions of our model with those observed for rocks and soils.
\end{abstract}

\section{Introduction}

Understanding multi-phase flow processes in porous rocks and soils is vital for addressing a number of problems in geosciences such as oil and gas recovery or vadose zone processes, which influence groundwater recharge and evaporation. Effective permeability, which is defined as the permeability of a fluid in the presence of another fluid, is the decisive parameter for fluid transport, and depends on fluid saturation, wetting condition, and pore structure. In addition, saturation history influences the fluid content and the effective permeability (for a specific pressure), which are different for imbibition and drainage.

A method considered suitable for determining water content of rocks non-invasively is nuclear magnetic resonance (NMR), because the NMR initial signal amplitudes are directly proportional to the hydrogen content in the pore space, and the NMR relaxation times are linked to the size of the water-containing pores in the rock. In a two-phase system of water and air, only the water contributes to the NMR signal response. Therefore, NMR is widely used for estimating transport and storage properties of rocks and sediments (Kenyon, 1997; Seevers, 1966; Fleury et al., 2001; Arnold et al., 2006).

In recent years, several researchers have studied the relationship between NMR and multiphase flow behavior on the pore scale to better understand and infer the storage and transport properties of partially saturated rocks or sediments (e.g., Chen et al., 1994; Liaw et al., 1996; Ioannidis et al., 2006; Jia et al., 2007; Al-Mahrooqi et al., 2006; Costabel and Yaramanci, 2011, 2013; Talabi et al., 2009). As an extension of this research, we study the relationship between the water distribution inside the pores of a partially satu- 


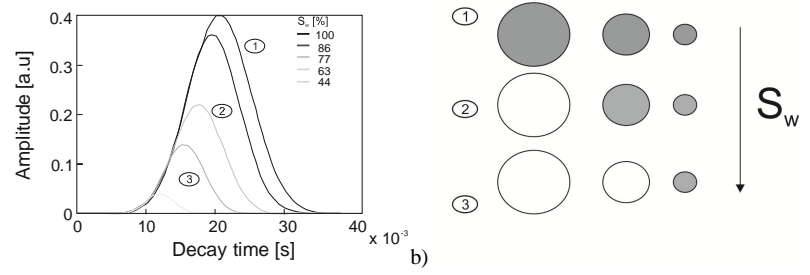

Figure 1. (a) NMR decay time distributions at different water saturation levels for a classical cylindrical capillary pore distribution. (b) Concept sketch of saturated (gray) and desaturated capillaries, e.g., during drainage.

rated rock and the system's NMR response by using bundles of pores with triangular cross sections. While Al-Mahrooqi et al. (2006) used a similar modeling approach to infer the wettability properties in oil-water systems, this study investigates the evolution of the NMR relaxation-time spectra during drainage and imbibition. For this purpose, we consider a capillary pore ensemble that is partially saturated with water and air. Traditionally, the pores within this ensemble are assumed to have a cylindrical geometry. Depending on pressure, cylindrical capillaries are either water- or air-filled, and thus they either contribute to an NMR response or not. Consequently, the NMR relaxation times of partially watersaturated capillary pore bundles always remain subsets of the fully saturated system's relaxation-time distribution; i.e., they are a function inside the envelope of the distribution curve at full saturation (see Fig. 1). However, in porous rocks, which are formed by the aggregation of grains, the pore geometry is usually more complex (Lenormand et al., 1983; Ransohoff and Radke, 1987; Dong and Chatzis, 1995) and may exhibit angular and slit-shaped pore cross sections rather than cylindrical capillaries or spheres (Fig. 2a). For example, in tight gas reservoir rocks, Desbois et al. (2011) found three types of pore shapes that are controlled by the organization of clay sheet aggregates: (i) elongated or slit-shaped, (ii) triangular, and (iii) multi-angular cross sections. The relaxationtime distribution functions derived from NMR measurements for such partially saturated rocks are frequently found to be shifted towards shorter relaxation times outside the original envelope observed for a fully saturated sample (Fig. 2b) (e.g., Applied Reservoir Technology Ltd., 1996; Bird et al., 2005; Jaeger et al., 2009; Stingaciu, 2010; Stingaciu et al., 2010; Costabel, 2011).

In angular pores, water will remain trapped inside the pore corners even if the gas entry pressure is exceeded. Standard NMR pore models that assume cylindrical or spherical pore ensembles (e.g., Kenyon, 1997), however, do not account for such residual water (Blunt et al., 2002; Tuller et al., 1999; Or and Tuller, 2000; Tuller and Or, 2001; Thern, 2014). To overcome this limitation, we adopt a NMR modeling approach initially proposed and discussed by Costabel (2011) and present numerical simulations and analytical solutions
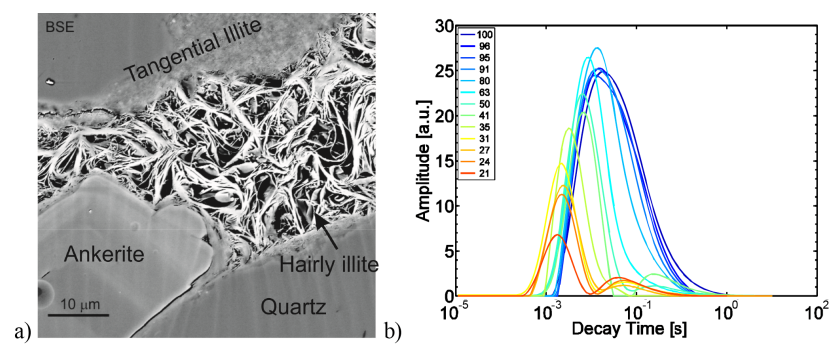

Figure 2. (a) Complex pore structure of a Rotliegend tight gas sandstone. Pore spaces are filled with tangential and hairly illite and exhibit different pore types with elongated or slit-shaped, triangular, and multi-angular cross sections. (b) $T_{1}$ decay time distributions calculated from inverse Laplace transform performed on Rotliegend sandstone (porosity $13 \%$, permeability $0.1 \mathrm{mD}$ ) at different water saturations $\left(S_{\mathrm{W}}=21 \%-100 \%\right)$.

of the NMR equations for partially saturated pores with triangular cross sections to quantify NMR signal amplitudes and relaxation times. The NMR response of a triangular capillary during drainage and imbibition depends on the water distribution inside the capillary, which is subject to pore shape and capillary pressure. Thus, in the next chapter, we present the relationship between capillary pressure and water distribution inside cylindrical and triangular pore geometries during drainage and imbibition. For this purpose, the reduced similar geometry concept introduced by Mason and Marrow (1991) is used. Subsequently, based on the spatial water distribution, an analytical solution of the NMR diffusion equation (Torrey, 1956; Brownstein and Tarr, 1979) for partially saturated triangular capillaries is derived and tested by numerical simulations (Mohnke and Klitzsch, 2010). The derived equations are used to study the influence of pore size distribution and pore shape of triangular capillaries on the NMR response, in particular considering the effects of trapped water. Finally, an approach for simulating NMR signals during imbibition and drainage of triangular pore capillaries is introduced and demonstrated using synthetic pore size distributions.

\section{Results and discussion}

\subsection{Water distribution during drainage and imbibition in a partially saturated triangular tube}

In a partially saturated pore space, a curved liquid-vapor interface called the arc meniscus (AM) arises due to the pore's capillary forces. In addition, adsorptive forces between water and matrix lead to the formation of a thin water film at the rock-air interface. Such water films with a thickness typically below $20 \mathrm{~nm}$ (e.g., Toledo et al., 1990; Tokunaga and Wan, 1997) exhibit very short NMR relaxation times. Although water films to some extent may influence transport properties and water distribution of a partially saturated 


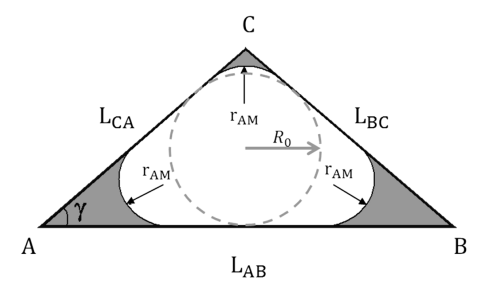

a)

Figure 3. Cross sections of a partially saturated triangular tube. The arc meniscus of radius $r_{\mathrm{AM}}$ separates the invading non-wetting phase (white) from the adsorbed wetting phase (gray). (a) Original triangle $\mathrm{ABC}$ with side lengths $L_{\mathrm{AB}}, L_{\mathrm{BC}}, L_{\mathrm{CA}}$, and radius $R_{0}$ of its inscribing circle. (b) Reduced triangle $\mathrm{A}^{\prime} \mathrm{B}^{\prime} \mathrm{C}^{\prime}$ of similar geometry. The wetting phase resides in the three corners (gray) with $r^{\prime}=r_{\mathrm{AM}}$ being the radius of both the three interface arc menisci of $\mathrm{ABC}$ and of the inscribing circle of $\mathrm{A}^{\prime} \mathrm{B}^{\prime} \mathrm{C}^{\prime}$.

porous system (Tuller and Or, 2001), the contribution of the film volume to NMR amplitudes is very small with respect to the NMR signal amplitudes arising from the water trapped in the menisci; i.e., $V_{\text {film }} \ll V_{\text {meniscus. }}$ Therefore, for the sake of simplicity, we neglect water films in this study.

In the following discussion, we consider a triangular capillary, initially filled with a perfectly wetting liquid, i.e., contact angle $\theta=0^{\circ}$, which exhibits a constant interfacial tension $\sigma\left(\sigma_{\text {air-water }}=73 \times 10^{-3} \mathrm{Nm}^{-1}\right.$ at $\left.20^{\circ} \mathrm{C}\right)$ and is under the assumption that gravity forces are weak and therefore can be neglected. The two-phase capillary entry pressure as derived by the MS-P method (Mayer and Stowe, 1965; Princen, 1969a, b, 1970) can be expressed by the Young-Laplace equation:

$p_{\mathrm{c}}=\frac{\sigma \cos \theta}{r_{\mathrm{AM}}}=\frac{\sigma}{r_{\mathrm{AM}}}$,

where $r_{\mathrm{AM}}$ is the radius of the interface arc meniscus and $p_{\mathrm{c}}$ is the minimum pressure difference necessary for a nonwetting phase, i.e., air, to invade a uniformly wetted (tri)angular tube filled with a denser phase, i.e., water (see Fig. 3a). Upon consideration of a pressure difference $p>p_{\mathrm{c}}$, the non-wetting phase will begin to enter the pore and occupy the central portion of the triangle, whereas - separated by the three interface arc menisci of radius $r_{\mathrm{AM}}$ - the wetting fluid remains in the pore corners (Fig. 3a).

From an original triangle $\mathrm{ABC}$, a new smaller triangle $\mathrm{A}^{\prime} \mathrm{B}^{\prime} \mathrm{C}^{\prime}$ of similar geometry with an inscribed circle of radius $r^{\prime}=r_{\mathrm{AM}}<R_{0}$ can be constructed by means of the reduced similar geometry concept as introduced by Mason and Morrow (1991) (Fig. 3b). To account for different transport mechanisms during imbibition and drainage of the denser wetting phase, Mason and Morrow (1991) introduced two different principal displacement curvatures with radii $r_{\mathrm{I}}$ and $r_{\mathrm{D}}$, respectively.

During imbibition of a (tri-)angular pore, the radius of curvature $r_{\mathrm{AM}}$ increases until the separate arc menisci of the cor- ners touch and the pore fills spontaneously ("snap off"). The critical radius of curvature $r_{\mathrm{I}}$, which is equal to the radius of the pore's inscribing circle, for the angular pore at "snap-off" pressure $p_{\mathrm{I}}$ is then given by

$r_{\mathrm{I}}=\frac{2 A}{P}$.

According to Eq. (2), the snap-off pressure depends on the geometry of the triangle only, i.e., on its cross-sectional area $A$ and perimeter $P$. In contrast, during drainage the threshold radius of curvature $r_{\mathrm{D}}=r_{\mathrm{AM}}$, at which the center of the fully saturated angular capillary spontaneously empties as the nonwetting fluid phase invades the pore, is given by

$r_{\mathrm{D}}=P\left[\frac{1}{2 G}+\left(\frac{\pi}{G}\right)^{1 / 2}\right]^{-1}$,

with $r_{\mathrm{D}}<r_{\mathrm{I}}$ and drainage threshold pressure $p_{\mathrm{D}}>p_{\mathrm{I}}$. The dimensionless and size-independent factor $G=\frac{A}{P^{2}}\left(=\frac{A^{\prime}}{P^{\prime 2}}\right)$ reflects the shape of the triangle, depending on its crosssectional area $A$ and perimeter $P\left(A^{\prime}\right.$ and $P^{\prime}$ refer to the reduced triangle), i.e., from near-slit shape $(G=0)$ to equilateral shape $(G=0.048)$. A detailed derivation of Eqs. (2) and (3) as a consequence of hysteresis between drainage and imbibition can be found in Mason and Morrow (1991).

The permeability of a porous system of such triangular capillaries is strongly influenced by the shape factor $G$. For single-phase laminar flow in a triangular tube, the hydraulic conductance $g$ is given by the Hagen-Poiseuille formula

$g=k \frac{A^{2} G}{\mu}$,

with the cross-sectional area $A$, the shape factor $G$, the fluid viscosity $\mu$, and $k$ being a constant accounting for the geometrical shape of the cross section; e.g., $k=0.5$ for circular tubes and $k=0.6$ for a tube with a cross section of an equilateral triangle (Patzek and Silin, 2001). The hydraulic conductance of an irregular triangle is closely approximated by Eq. (1) using the same constant $k$ as for an equilateral triangle (Øren et al., 1998). Thus, for a constant cross-sectional area, the hydraulic conductance $g$ of the pore is proportional to its shape factor $G$.

Combining Eqs. (1)-(3) with the concept of reduced similar geometry discussed above, the degree of water saturation $\left(S_{\mathrm{w}}\right)$ inside a single triangular tube with cross-sectional area $A_{0}$, perimeter $P_{0}$, and radius $R_{0}$ of its inscribing circle at a given capillary pressure $p_{\mathrm{c}}$ during imbibition and drainage 

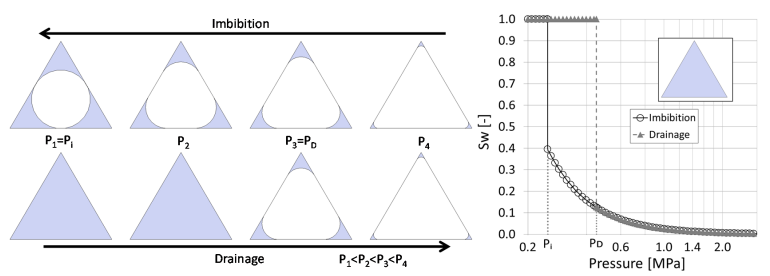

b)

Figure 4. (a) Modeled distribution of water (gray) and gas (white) phases in an equilateral triangular tube with a side length of $1 \mu \mathrm{m}$ during imbibition (top) and drainage (bottom). (b) Water saturation versus capillary pressure during imbibition $(\bigcirc)$ and drainage $(\boldsymbol{\Delta})$.
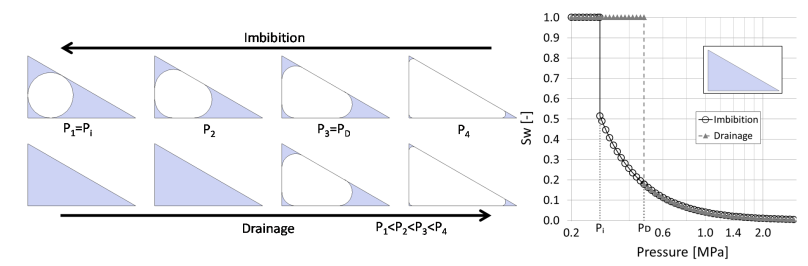

a)

b)

Figure 5. (a) Modeled distribution of water (gray) and gas (white) phases in a right-angled triangular capillary $(G=0.39)$ with side lengths $L=1,0.81,0.58 \mu \mathrm{m}$, and perimeter $P=2.39 \mu \mathrm{m}$ during imbibition (top) and drainage (bottom). (b) Water saturation versus capillary pressure during imbibition $(\bigcirc)$ and drainage $(\boldsymbol{\Lambda})$.

can be calculated according to

$S_{\mathrm{W}}^{\mathrm{I}}\left(p, A_{0}, P_{0}\right)=$

$\left\{\begin{array}{ll}1, & p_{\mathrm{c}} \leq p_{\mathrm{I}}\left(R_{0} \leq r_{\mathrm{I}}\right) \\ \frac{A_{\Delta}\left(p_{\mathrm{c}}\right)}{A_{0}}, & p_{\mathrm{c}}>p_{\mathrm{I}}\left(R_{0}>r_{\mathrm{I}}\right)\end{array}\right.$ (imbibition)

$S_{\mathrm{w}}^{\mathrm{D}}\left(p_{\mathrm{c}}, A_{0}, P_{0}\right)=$

$\left\{\begin{array}{ll}1, & p_{\mathrm{c}}<p_{\mathrm{D}}\left(R_{0}<r_{\mathrm{D}}\right) \\ \frac{A_{\Delta}\left(p_{\mathrm{c}}\right)}{A_{0}}, & p_{\mathrm{c}} \geq p_{\mathrm{D}}\left(R_{0} \geq r_{\mathrm{D}}\right)\end{array}\right.$ (drainage)

The total area $A_{\Delta}$ of the triangular tube's water retaining corners, $\gamma_{1,2,3}$ (i.e., the gray areas in Figs. 4 and 5), is expressed by

$A_{\Delta}\left(p_{\mathrm{c}}\right)=\sum_{i=1}^{3} A_{\gamma_{i}}\left(p_{\mathrm{c}}\right)$

where

$$
\begin{aligned}
A_{\gamma_{i}}\left(p_{\mathrm{c}}\right) & =\left(\frac{1}{\tan \frac{\gamma_{i}}{2}}-\frac{\left(\pi-\gamma_{i}\right)}{2}\right) r_{\mathrm{AM}}^{2}\left(p_{\mathrm{c}}\right) \\
\text { and } & 0<\gamma_{i}<\pi
\end{aligned}
$$

is the area of the triangle's $i$ th water-filled corner (Tuller and Or, 2001). Consequently, the total effective area $A_{\Delta}$ that is still occupied by water is equal to the difference between the (reduced) triangular pore area $\widetilde{A}$ and the area $\pi r_{\mathrm{AM}}^{2}$ of its respective inscribing circle (see Fig. 3). The above Eq. (7a) + (b) can be simplified to $A_{\Delta}=$ $(3 \sqrt{3}-\pi) r_{\mathrm{AM}}\left(p_{\mathrm{c}}\right)$ when considering equilateral triangles, i.e., $\gamma_{1,2,3}=\frac{\pi}{3}$. The radius $r_{\mathrm{AM}}\left(p_{\mathrm{c}}\right)$ of the reduced triangle's arc meniscus can be directly calculated from Eq. (1). Calculated pressure-dependent water and gas distributions during imbibition and drainage for an equilateral and arbitrary triangular capillary are shown in Figs. 4a and 5a. The corresponding water retention curves plotted in Figs. $4 \mathrm{~b}$ and $5 \mathrm{~b}$ illustrate the resulting hysteresis behavior of the partially saturated system and can be subdivided into three parts: at low capillary pressures, i.e., $p_{\mathrm{c}}<p_{\mathrm{I}}$, where the pore always remains fully water-saturated. For the interval $p_{\mathrm{I}}<p_{\mathrm{c}} \leq p_{\mathrm{D}}$ where two separate behaviors are observed: during imbibition, the water content gradually increases with increasing capillary pressure, while during drainage the pore still remains fully saturated. For pressure levels $p_{\mathrm{c}} \geq p_{\mathrm{D}}$, both drainage as well as imbibition exhibit the same gradual decrease in water saturation.

In the following section, analytical solutions for respective NMR responses that arise from partially saturated arbitrary triangular tubes are derived and matched against numerical simulations by means of the generalized differential NMR diffusion equations introduced by Brownstein and Tarr (1979).

\subsection{NMR response for triangular capillaries}

The measured NMR relaxation signal $M(t)$ is constituted by superposition of all signal-contributing pores in a rock sample (e.g., Coates et al., 1999; Dunn et al., 2002):

$\frac{M(t)}{M_{0}}=\frac{1}{V_{0}} \sum_{i}^{N}\left(v_{i} \times\left(1-e^{t \cdot T_{i, 1}^{-1}}\right)\right)$,

where $M_{0}$ and $V_{0}$ are the equilibrium magnetization and total volume of the pore system, respectively. The saturated volume of the $i$ th pore and its corresponding longitudinal relaxation constant are given by $v_{i}$ and $T_{i, 1}$, respectively.

Following derivations of Brownstein and Tarr (1979), the inverse of the longitudinal relaxation time $T_{1}$ is linearly proportional to the surface-to-volume ratio of a pore according to

$T_{1}^{-1}=T_{1 \mathrm{~B}}^{-1}+\rho_{\mathrm{s}} \frac{S_{\mathrm{a}}}{V}$,

where $T_{1 \mathrm{~B}}$ is the bulk relaxation time of the free fluid and $\rho_{\mathrm{s}}$ is the surface relaxivity, a measure of how quickly protons lose their magnetization due to magnetic interactions with paramagnetic impurities and reduced correlation times at the fluid-solid interface, which can be attributed to paramagnetic ions at mineral grain surfaces. $V$ and $S_{\text {a }}$ are the pore's volume and active surface boundaries, respectively. In this context, 


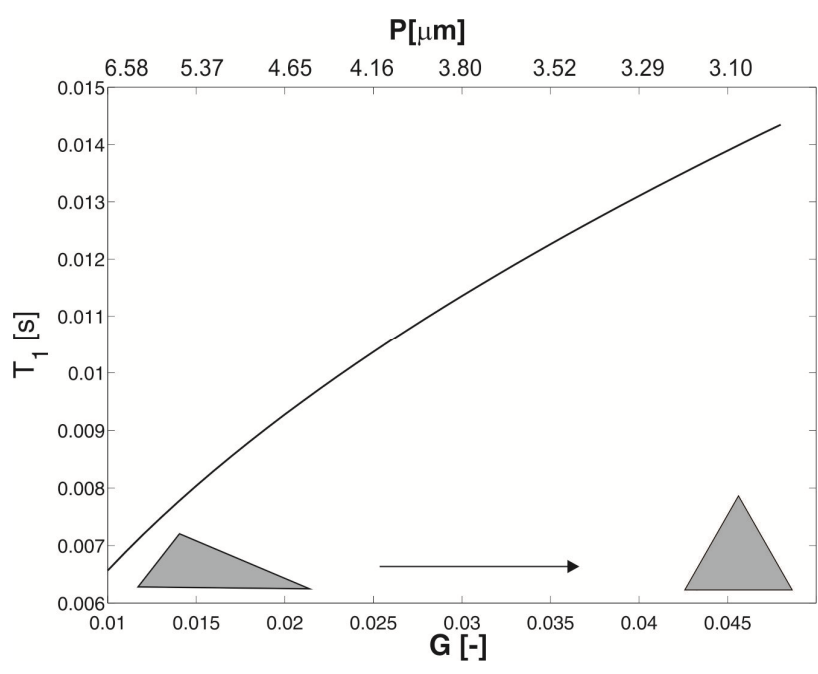

Figure 6. Longitudinal relaxation times $T_{1}$ of fully saturated triangular pores with constant cross-sectional area $A=4.33 \times 10^{-13} \mathrm{~m}^{2}$ versus shape factor $G=\frac{A}{P^{2}}$ and perimeter $P$. NMR parameters: $\rho_{\mathrm{s}}=10 \mu \mathrm{ms}^{-1}, T_{1 \mathrm{~B}}=3$.

an active boundary refers to an interfacial area, i.e., the pore wall, where $\rho_{\mathrm{s}}>0$ and, thus, enhanced NMR relaxation will occur as the molecules diffuse at the pore walls. This model, however, is based on the general assumption of a relaxation regime that is dominated by surface relaxation processes (fast diffusion); i.e., the fluid molecules move sufficiently quickly and thus explore all parts of the pore volume several times with respect to the timescale $\left(\sim T_{1}\right)$ of the experiment.

Upon consideration of a long (triangular) capillary, its surface-to-volume ratio equals its perimeter-to-cross-section ratio, i.e., $S / V=P / A$. Consequently, Eq. (9) can be written as

$T_{1}^{-1}=T_{1 \mathrm{~B}}^{-1}+\rho_{s} \frac{P_{0}}{A_{0}}$,

where $P_{0}$ is the saturated tube's (active) perimeter and $A_{0}$ its cross-sectional area for a circular cross section, $\frac{P_{0}}{A_{0}}=\frac{2}{r_{0}}$, with $r_{0}$ being the capillary radius. Hence, the relaxation rate of a fully saturated arbitrary triangular pore $\mathrm{ABC}$ can be expressed in terms of its shape factor $G$ and perimeter $P_{0}$ :

$T_{1}^{-1}=T_{1 \mathrm{~B}}^{-1}+\frac{\rho_{s}}{G P_{0}}\left(=T_{1 \mathrm{~B}}^{-1}+\rho_{s} \frac{L_{\mathrm{AB}}+L_{\mathrm{BC}}+L_{\mathrm{CA}}}{L_{\mathrm{AB}} L_{\mathrm{CA}} \sin ?\left(\gamma_{\mathrm{A}}\right)}\right)$,

where $L_{\mathrm{AB}}, L_{\mathrm{BC}}$, and $L_{\mathrm{AC}}$ are the lengths of a triangle's sides and $\gamma_{A}$ is the angle at corner $A$ (see Fig. 3). As illustrated in Fig. 6 , the relaxation times of a fully saturated pore decrease with decreasing pore shape factor $G$ - and thus, decreasing hydraulic conductance - and increasing pore perimeter $P$. By reducing one angle from 60 to $0^{\circ}$ while fixing another at $60^{\circ}$, we increase $P / A$ for a constant cross-sectional area $A$. In the special case of an equilateral triangular capillary, i.e.,

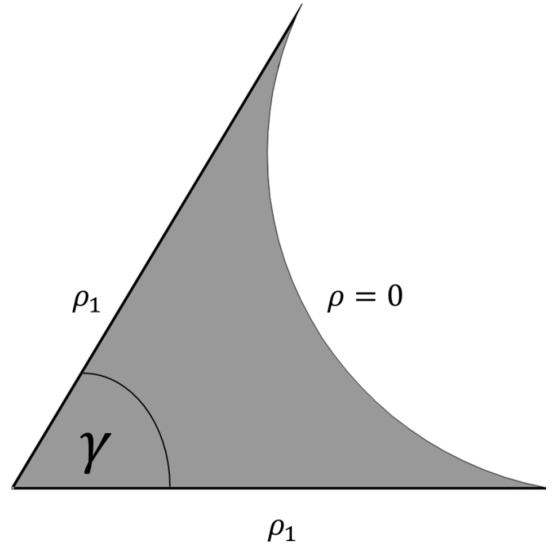

Figure 7. Saturated corner with active boundaries, i.e., $\rho_{\mathrm{s}}=\rho_{1}>0$ at the corner's perimeter $P_{\gamma}$ and a passive boundary at the air-water interface (meniscus), i.e., $\rho_{\mathrm{s}}=\rho=0$.

$P_{0} / A_{0}=\frac{12}{\sqrt{3} L_{0}}$, Eq. (11) can be simplified to

$T_{1}^{-1}=T_{1 \mathrm{~B}}^{-1}+\rho_{\mathrm{s}} \frac{12}{\sqrt{3} L_{0}}$.

Now we consider the previously discussed water-air system of a partially saturated equilateral triangular capillary. Here, the NMR signal will originate from the water retained at the corners, replacing $A_{0}$ in Eq. (10) with an effective area $A_{\gamma}$ or $A_{\Delta}$ as derived by Eqs. (7a) and (b), respectively. $A_{\Delta}$ reflects the actual pore fraction that contributes to the NMR signal, i.e., the portion of the pore area $A_{0}$ that still remains occupied by water.

Supposing the air-water interface to be a passive boundary with respect to NMR surface relaxivity, i.e., $\rho_{\mathrm{S}}=0$, the effective active boundary is exclusively controlled by the pore wall segments $\left(\rho_{\mathrm{S}}>0\right)$ in contact with water (wetting phase) (Fig. 7). Thus, the active perimeter of such a partially saturated triangular capillary is equal to its pressure-dependent reduced triangle's perimeter, $P_{\Delta}^{\prime}\left(r^{\mathrm{I}, \mathrm{D}}\left(p_{\mathrm{c}}\right)\right)$, according to

$P_{\Delta}=\sum_{i=1}^{N=3} P_{\gamma_{i}}$

with

$P_{\gamma_{i}}=2 \frac{r_{\mathrm{AM}}\left(p_{\mathrm{c}}\right)}{\tan \frac{\gamma_{i}}{2}} 0<\gamma_{i}<\pi$

being the perimeter of the $i$ th water-filled corner. Consequently, the NMR relaxation rates and NMR signal (amplitude) evolution during drainage and imbibition of a single equilateral triangular capillary can be expressed by

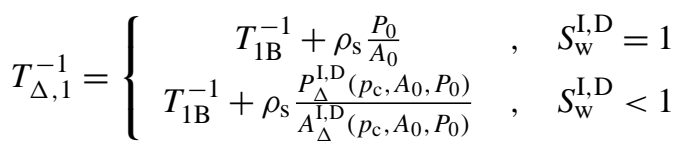



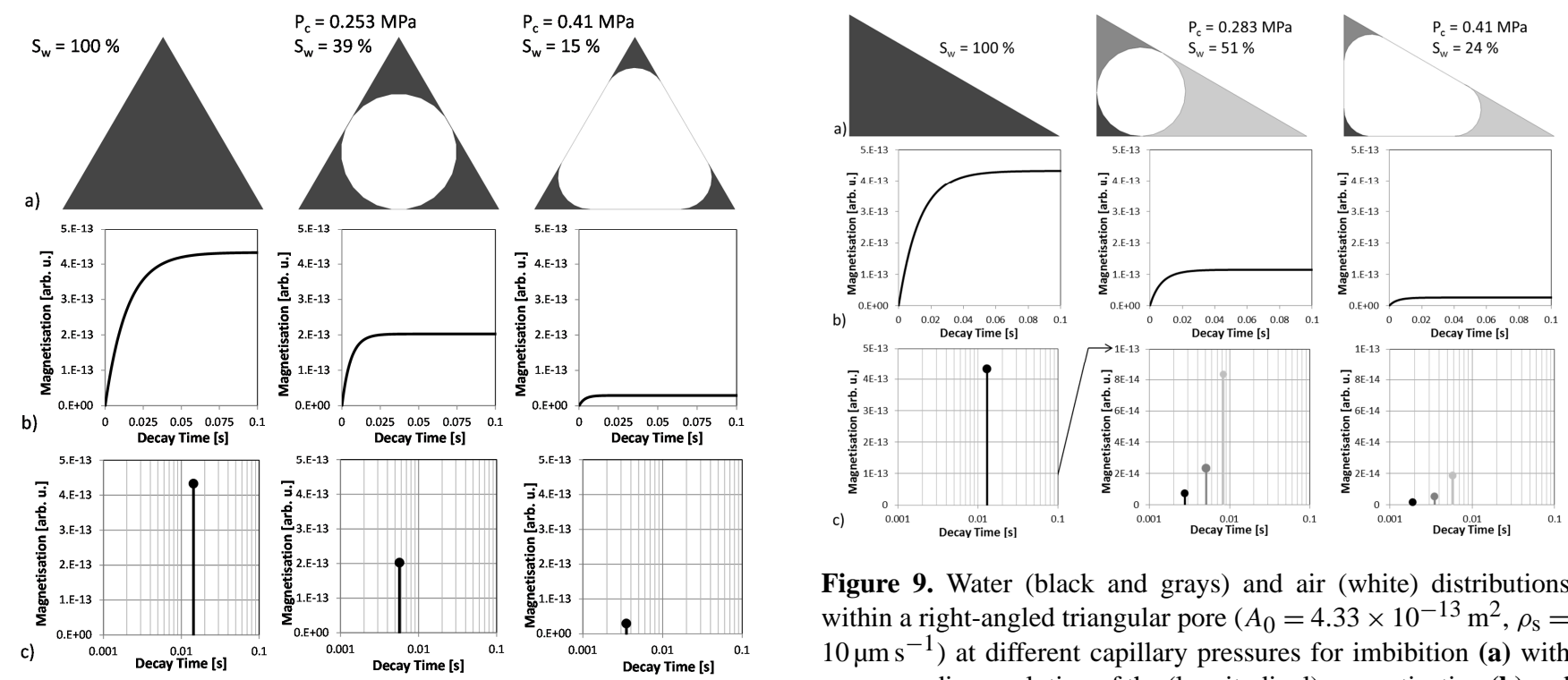

Figure 9. Water (black and grays) and air (white) distributions within a right-angled triangular pore $\left(A_{0}=4.33 \times 10^{-13} \mathrm{~m}^{2}, \rho_{\mathrm{s}}=\right.$ $10 \mu \mathrm{m} \mathrm{s}^{-1}$ ) at different capillary pressures for imbibition (a) with corresponding evolution of the (longitudinal) magnetization (b) and

Figure 8. Water (black) and air (white) distributions within a triangular pore $\left(A_{0}=4.33 \times 10^{-13} \mathrm{~m}^{2}, \rho_{\mathrm{s}}=10 \mu \mathrm{m} \mathrm{s}^{-1}\right)$ at different capillary pressures for imbibition (a) with corresponding evolution of the (longitudinal) magnetization (b) and NMR $T_{1}$ relaxation times (c).

and

$\frac{m(t)}{m_{0}}=S_{\mathrm{w}}^{\mathrm{I}, \mathrm{D}}\left(p_{\mathrm{c}}, A_{0}, P_{0}\right)\left(1-e^{\frac{-t}{T_{\Delta, 1}}}\right)$,

respectively. Figure 8 illustrates the pressure-dependent water distribution inside a single equilateral triangular capillary (with a side length of $1 \mu \mathrm{m}$ ) during drainage (a) and evolution of longitudinal magnetization (b). As the water saturation is reduced with increasing pressure, both NMR amplitudes and relaxation times (c) decrease. Note that only a single characteristic relaxation time at each saturation degree is observed, since each corner has the same $P_{\gamma} / A_{\gamma}$ and consequently the same $T_{1}$ value.

In contrast, each water-filled corner of a partially saturated non-equilateral triangle, i.e., $\gamma_{1} \neq \gamma_{2} \neq \gamma_{3}$, can have a different $P_{\gamma} / A_{\gamma}$ ratio, and thus will show a different relaxation time and amplitude. As a result, depending on its individual shape, even a single partially saturated pore exhibits a multiexponential NMR relaxation behavior based on Eq. (8) according to

$\frac{m(t)}{m_{0}}=\frac{1}{A_{0}} \sum_{i=1}^{N=3} A_{\gamma_{i}}^{\mathrm{I}, \mathrm{D}}\left(1-e^{\frac{-t}{T_{\gamma_{i}}, 1}}\right)$

with $T_{\gamma_{i, 1}}=\frac{1}{T_{1 \mathrm{~B}}}+\rho_{\mathrm{s}} \frac{P_{\gamma_{i}}}{A_{\gamma_{i}}}$ and $\frac{A_{\gamma_{i}}^{\mathrm{I}, \mathrm{D}}}{A_{0}}$ being the characteristic relaxation time and amplitude contribution of the $i$ th corner of the triangle, respectively. Figure 9 exemplifies such different multi-exponential relaxation behavior for a pore with a right triangle geometry with angles of $\left(\gamma_{1}=30^{\circ}, \gamma_{2}=60^{\circ}\right.$, 


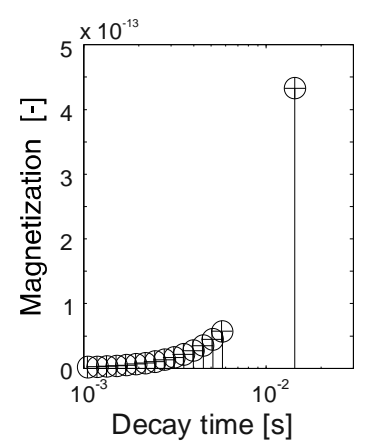

a)

Figure 10. NMR response of an equilateral triangular capillary pore model (with a side length of $1 \mu \mathrm{m}$ ). (a) Magnetization versus $T_{1}$ decay time data of numerical $(\bigcirc)$ and analytical solutions $(+)$ for all applied pressure levels. (b) Cross-plot of numerically simulated and analytically calculated longitudinal $T_{1}$ decay times at partial $(\bullet)$ and full water saturation $(\boldsymbol{\square})$. A corresponding water saturation versus capillary pressure diagram is shown in Fig. 4.

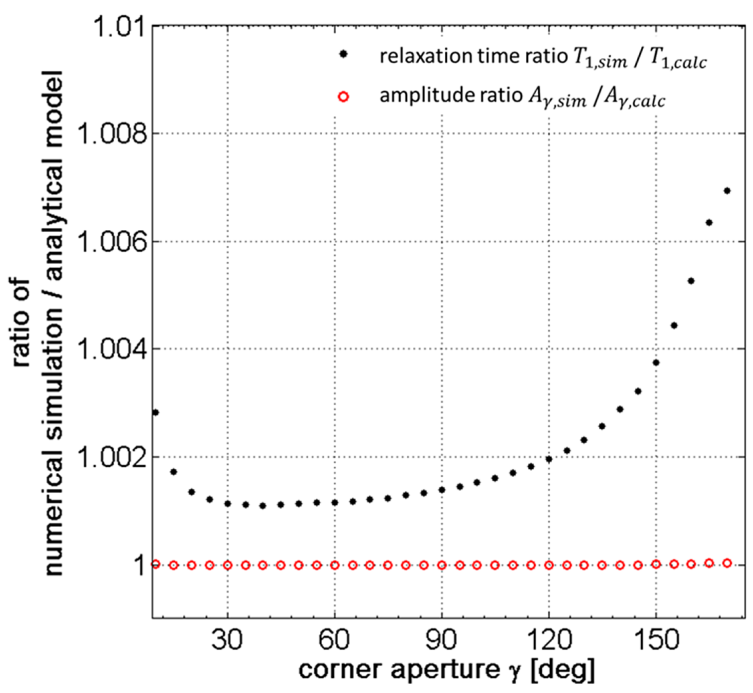

Figure 11. Comparison of analytical and calculated NMR relaxometry data originating from saturated pore corners (e.g., see Fig. 7) of varying apertures $\left(5^{\circ}<\gamma<175^{\circ}\right)$ and an equal active surfaceto-volume ratio $\frac{P_{\gamma_{i}}}{A_{\gamma_{i}}}=$ const (NMR model parameters: $T_{1 \mathrm{~B}}=3 \mathrm{~s}$, $\left.D=2.5 \times 10^{-9} \mathrm{~m}^{2} \mathrm{~s}^{-1}, \rho_{\mathrm{s}}=10 \mu \mathrm{m} \mathrm{s}^{-1}\right)$.

simulations were compiled and compared to their respective analytical solutions. The ratios of the numerical to the analytical model results for NMR amplitudes, i.e., NMR signal amplitudes, $A_{\gamma}$, and relaxation times, $T_{1, \gamma}$, as a function of corner aperture $\gamma$ are shown and confirm a near-perfect correlation of $R^{2}>0.99$, with deviations generally less than $0.05 \%$. In this regard, the slight increase in the divergence of relaxation-time ratios at acute and obtuse angles can be attributed to numerical errors resulting from a decrease in the finite element's grid quality due to extremely high or low $x$ -

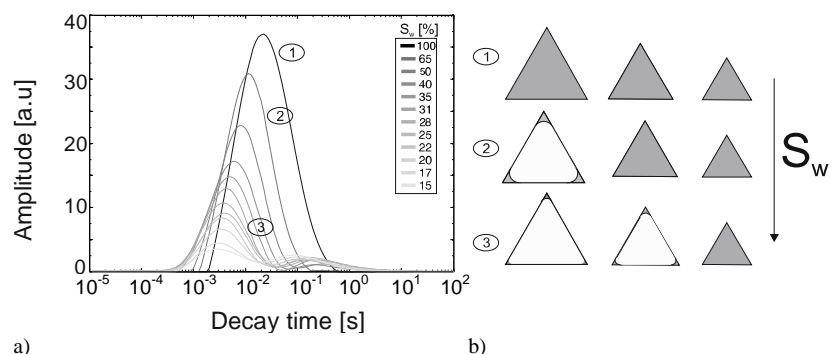

Figure 12. (a) NMR decay time distributions at different water saturation levels for a pore distribution of equilateral triangles. (b) Concept sketch of saturated (gray) and desaturated triangular capillaries for increasing pressure levels (1), (2) and (3), e.g., during drainage.

to- $y$ ratios at these apertures. The above model is applicable to any angular capillary geometry, such as square or octahedron.

\subsection{Simulated water retention curves and NMR relaxation data of partially saturated pore distributions}

The goal of this section is to evaluate how pore shape affects the forward-modeled NMR response of a partially saturated system of pores (a pore size distribution). As discussed earlier, the NMR relaxation time of a single water-filled capillary pore is inversely proportional to its surface-to-volume ratio. Thus, at full water saturation, the relaxation-time distribution obtained from a multi-exponential NMR relaxation signal represents the pore size distribution of the rock. At partial water saturation it is often assumed that the NMR relaxation signal still represents the pore size distribution of the water-saturated pores (e.g., Stingaciu et al., 2010). We are going to demonstrate that this is valid for cylindrical but not for (tri-)angular pores.

In contrast to cylindrical pores, capillaries with (tri)angular cross sections may be partially water-saturated during drainage or imbibition (cf. Figs. 8 and 9) because of the water remaining in the corners. Thus, they show a different water retention behavior, and the "desaturated" pores, i.e., their arc menisci, contribute to the NMR signal. Consequently, with increasing pressure (i.e., decreasing water saturation), the NMR relaxation behavior of the partially watersaturated triangular capillary pore bundle successively shifts to signal contributions with shorter relaxation times, exceeding the original distribution at full saturation. This shift reflects the fast relaxation of residual water trapped in the pore corners (Fig. 12). This behavior in angular pore geometries is demonstrated in Fig. 13. Here, the NMR relaxation components for a fully (blue line) and partially saturated (red and green) distribution of triangular capillaries are plotted. The green and red peaks show the signals of the residual water in the pore corners. As a consequence of the reduced geometry concept, the remaining water in the corners can be 


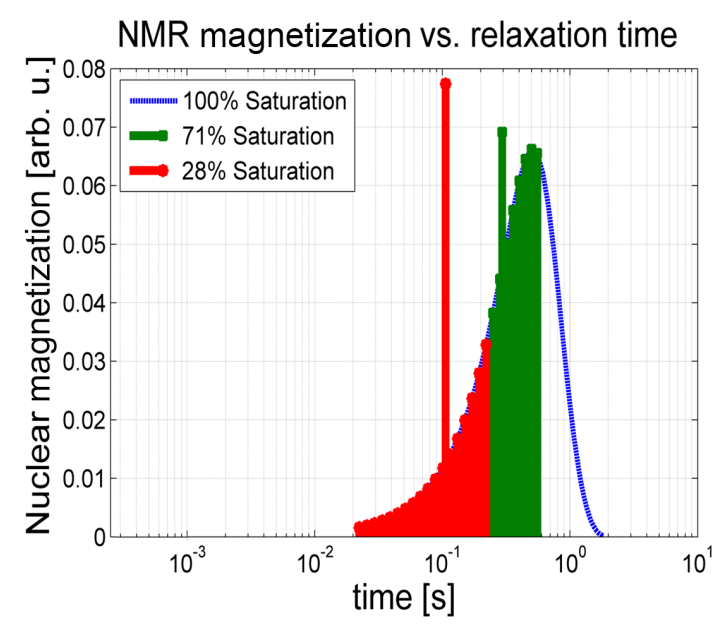

Figure 13. Relaxation components of fully (blue line) and partially desaturated triangular pore size distribution. At a specific saturation level, all pore corners with residual saturation exhibit the same NMR magnetization and relaxation behavior, thus superposing to a single fast relaxation component (e.g., red and green bars).

considered similar in size and shape due to the same NMR relaxation time, and thus only depends on pressure and not on pore size. Therefore, with decreasing saturation, i.e., increasing pressure, the NMR signal of the arc menisci increases and shifts towards smaller relaxation times. If the non-wetting phase (air) has entered all capillaries, only one single relaxation time remains for the pore bundle of equilateral triangles. For arbitrarily shaped triangular pores, three relaxation times would remain for the desaturated pore system. Hence, the concept of a relaxation-time distribution assumed in conventional NMR inversion and interpretation approaches would be no longer valid.

We applied the concept of fitting multi-exponential relaxation-time distributions to NMR transients calculated for pore bundles of circular and equilateral triangle cross sections in order to study how pore shape affects the typically shown relaxation-time distributions.

Water drainage and imbibition with water as the wetting and air as the non-wetting fluid were investigated by simulating water retention curves and corresponding NMR relaxation signals for a log-normal distributed pore size ensemble as shown in Fig. 14.

Herein, to clarify the subsequent discussion, we focused only on the equilateral triangular capillary model. Other angular pore shapes (e.g., right angular triangles or squares) will exhibit a similar behavior. Capillary pressure curves presented in Fig. 15a were calculated from Eqs. (1), (5), and (6) for pore bundles with circular and equilateral triangle cross sections. In contrast to water retention curves calculated for the cylindrical capillary model, significant hysteresis between drainage and imbibition can be observed for the triangular capillary model, i.e., in terms of initial am-

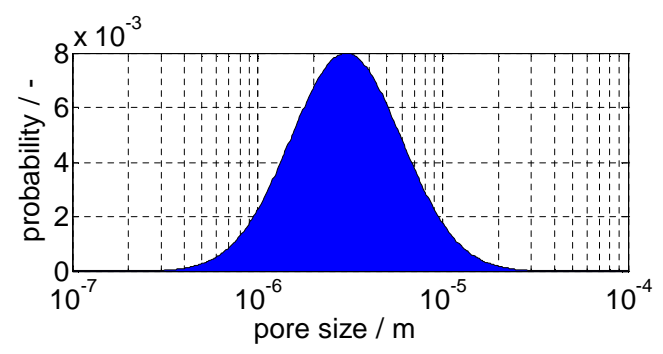

Figure 14. Pore size distribution model (log-normal distribution: $\sigma=0.3 \mu=3 \times 10^{-6} \mathrm{~m}$ in analogy to that of the Rotliegend sandstone shown in Fig. 2).

plitudes (= saturation) and respective mean relaxation times (Fig. 15b). Corresponding NMR $T_{1}$ relaxation (saturation recovery) signals shown in Fig. 15c, $d$ and e were calculated using a uniform surface relaxivity of $\rho_{\mathrm{s}}=10 \mu \mathrm{m} \mathrm{s}^{-1}$ and a water bulk relaxation of $T_{1, \text { bulk }}=3 \mathrm{~s}$.

The NMR $T_{1}$ relaxation signals were simulated for 20 saturation levels of the drainage and imbibition curves ranging from $S=100 \%$ to $S<1 \%$ water saturation. The corresponding relaxation-time distributions (Fig. $15 \mathrm{f}-\mathrm{h}$ ) of the NMR $T_{1}$ transients were determined by means of a regularized multi-exponential fitting using a nonlinear least squares formulation solved by the Levenberg-Marquardt approach (e.g., Marquardt, 1963; Mohnke, 2010). Inverse modeling results of NMR data calculated for the drainage branches using the cylindrical capillary bundle (Fig. 15f) exhibit a shift of the distribution's maximum towards shorter relaxation times with decreasing saturation (i.e., increasing pressure). As anticipated, the derived distribution functions remain inside the envelope of the relaxation-time distribution curve at full saturation (see also Fig. 1a).

In contrast, inversion results for equilateral triangular capillary ensembles (Fig. 15f-h) - both for imbibition and drainage - show a similar shift to shorter relaxation times with decreasing saturation but also shift towards the outside the initial distribution at full saturation due to NMR signals originating from trapped water in the pore corners of the desaturated triangular capillaries. The effect of the pore corners on relaxation times at low saturations is also recognizable when comparing the (geometric) mean relaxation times, normalized on the values observed at full saturation (Fig. 15b): both, the drainage and the imbibition hysteresis branch of the triangular pore bundle, show smaller mean relaxation times than the cylindrical pore bundle.

In conclusion, the calculated inverse models for the triangular capillary bundle qualitatively agree with the behavior of the inverted NMR relaxation-time distributions at partial saturation that are frequently observed in experimental data, e.g., of the Rotliegend sandstone shown in Fig. 2. 

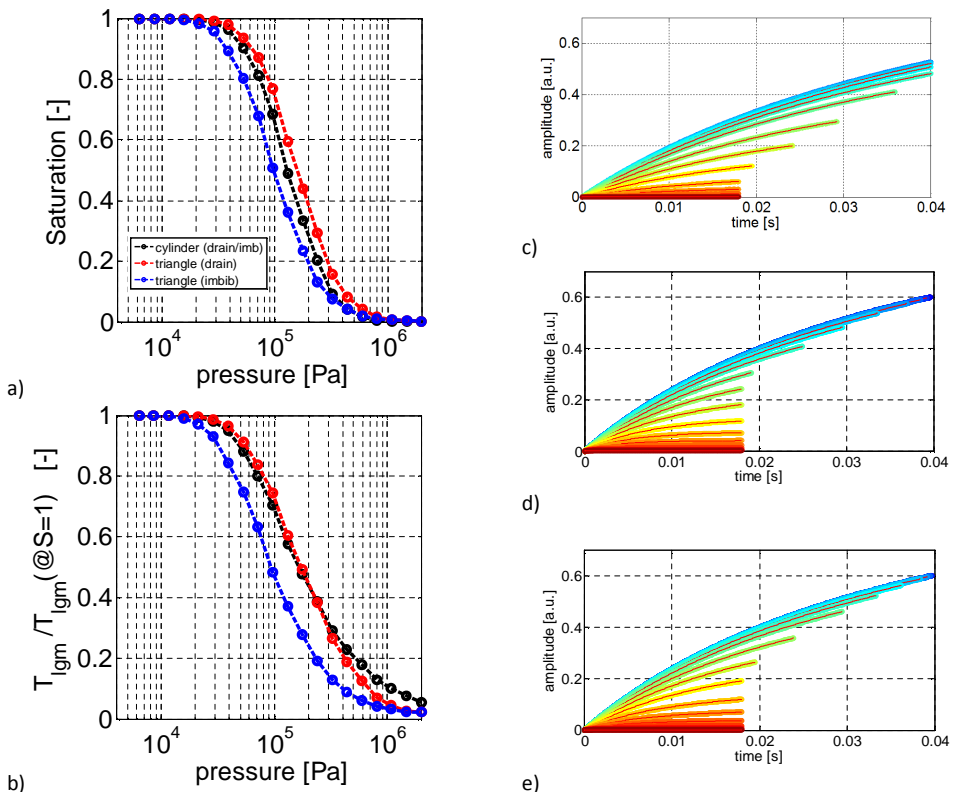

c)

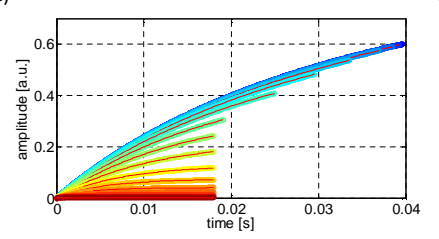

d)

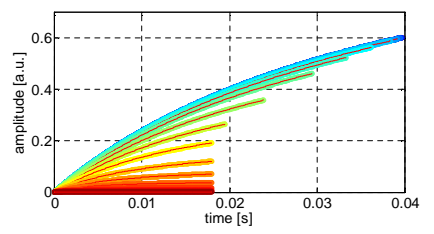

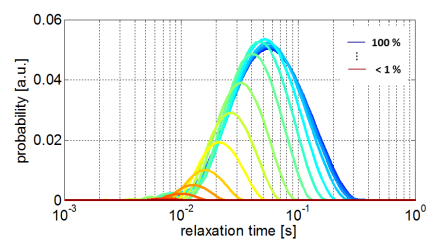
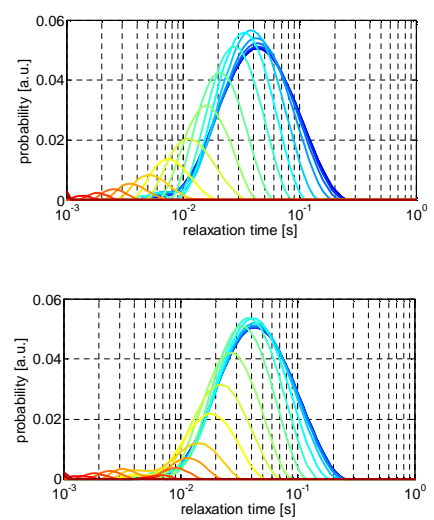

e)

Figure 15. (a) Modeled drainage and imbibition curves for circular and equilateral triangular capillary ensemble (cf. Fig. 14) and (b) Corresponding normalized mean NMR $T_{1}$ relaxation times versus pressure curves. Modeled and fitted (red lines) NMR transient signals (longitudinal magnetization evolution) and corresponding inverted NMR $T_{1}$ relaxation-time distributions for 20 fully and partially saturated pore size distributions ranging from $<1$ to $100 \%$ saturation using circular (c, f) and equilateral triangular capillaries during imbibition (d, g) and drainage (e, h).

\section{Summary and conclusions}

Experimental NMR relaxometry data and corresponding relaxation-time distributions obtained at partial water/air saturation were explicated by a modification of conventional NMR pore models using triangular cross sections. The derived analytical solutions for calculating surface-dominated (fast diffusion) NMR relaxation signals in fully and partially saturated arbitrary angular capillaries were consistent with respective results obtained from numerical simulations of the general NMR diffusion equations.

Shape and size of triangular pores can strongly influence both NMR amplitudes and decay time distribution and the rock's flow properties, i.e., saturation and (relative) permeability. At full saturation the NMR relaxation time depends on the surface-to-volume ratio, which in turn depends on shape if considering angular pore capillaries. However, at partial saturation, the pore shape even more strongly influences the water distribution inside the pore system, and thus the NMR signal. In contrast to cylindrical capillaries, angular capillaries also contribute to the NMR signal even after desaturation of the pore due to the water remaining in the pore corners.

In this regard, non-equilateral triangular capillaries at partial saturation exhibit a three-exponential relaxation behavior due to different perimeter-to-surface (= surface-to-volume) ratios of the water in the pore corners, whereas the relaxation time of the trapped water in the corners depends on pressure and not on pore size. Therefore, it can be noted that the NMR signal at partial saturation is affected by both the surface-tovolume ratio of the water saturated and the pore shape of the desaturated pores.

Moreover, we studied the NMR response of a triangular pore bundle model by jointly simulating the water retention curves for drainage and imbibition and the corresponding NMR $T_{1}$ relaxometry data. With decreasing water saturation, the simulated NMR relaxation distributions shift towards shorter relaxation times below the initial distribution enveloped at full saturation, which is principally in agreement with the relaxation behavior observed in experimental NMR data from rocks (e.g., Fig. 2b).

Ongoing research will include further experimental validation and implementation of the introduced approach in an inverse modeling algorithm for NMR data obtained from partially saturated rocks to predict absolute and relative permeability on laboratory and borehole scales. Without considering angular pores, the NMR signal of trapped water cannot be explained; i.e., using the classical approach of circular capillaries, one cannot find a pore size distribution that explains the relaxation-time distributions at all saturations sufficiently (e.g., Mohnke, 2014). On the other hand, angular pore models can account for the trapped water and thus overcome the limitation of the classical approach. Moreover, following the approach of Mohnke (2014) but considering angular pores, we strive to estimate surface relaxivity, pore size distribution, and pore shape by jointly inverting NMR data at differ- 
ent saturations. Based on the obtained pore size distribution and triangle shape, we expect to improve the prediction of the absolute and relative permeabilities considerably.

Acknowledgements. The study was supported by the German Research Foundation (DFG) in the framework of the Transregional Collaborative Research Centre 32 (SFB TR 32) and Wintershall AG in the framework of the Wintershall Tight Gas Consortium at RWTH Aachen University.

Edited by: M. Giudici

\section{References}

Al-Mahrooqi, S. H., Grattoni, C. A., Muggeridge A. H., Zimmermann, R. W., and Jing, X. D.: Pore-scale modelling of NMR relaxation for the characterization of wettability, J. Petrol. Sci. Eng., 52, 172-186, 2006.

Applied Reservoir Technology Ltd.: The NMR Sandstone Rock Catalogue, Long Melford, Suffolk, UK, 1996.

Arnold, J., Clauser, C., Pechnig, R., Anferova, S., Anferov, V., and Blümich, B.: Porosity and Permeability from Mobile NMR CoreScanning, Petrophysics, 47, 306-314, 2006.

Bird, N. R. A., Preston, A. R., Randall, E. W., Whalley, W. R., and Whitmore, A. P.: Measurement of the size-distribution of water-filled pores at different matric potentials by STRAFI-NMR relaxation-time measurements, Eur. J. Soil Sci., 56, 135-143, 2005.

Blunt, M. J., Jackson, M. D., Piri, M., and Valvatne, P. H.: Detailed physics, predictive capabilities and macroscopic consequences for pore-network models of multiphase flow, Adv. Water Resour., 25, 1069-1089, 2002.

Brownstein, K. and Tarr, C.: Importance of classical diffusion in NMR studies of water in biological cells, Phys. Rev. A, 19, 24462453, 1979.

Chen, S., Liaw, H. K., and Watson, A. T.: Measurements and analysis of fluid saturation-dependent NMR relaxation and linebroadening in porous media, Magn. Reson. Imaging, 12, 201-202, 1994.

Coates, G. R., Xiao, L., and, Prammer, M. G.: NMR Logging Principles and Applications, Halliburton Energy Services, Houston, TX, 234 pp., 1999.

Costabel, S.: Nuclear magnetic resonance on laboratory and field scale for estimating hydraulic parameters in the vadose zone, $\mathrm{PhD}$ thesis, Berlin University of Technology, available at: opus4. kobv.de/opus4-tuberlin/files/3173/costabel_stephan.pdf, 2011.

Costabel, S. and Yaramanci, U.: Relative hydraulic conductivity in the vadose zone from magnetic resonance sounding - BrooksCorey parameterization of the capillary fringe, Geophysics, 76, G61-G71, doi:10.1190/1.3552688, 2011.

Costabel, S. and Yaramanci, U.: Estimation of water retention parameters from nuclear magnetic resonance relaxation time distributions, Water Resour. Res., 49, 2068-2079, doi:10.1002/wrcr.20207, 2013.

Desbois, G., Urai, J. L., Kukla, P. A., Konstanty, J., and Baerle, C.: High-resolution 3D fabric and porosity model in a tight gas sandstone reservoir: A new approach to investigate microstructures from mm- to nm-scale combining argon beam crosssectioning and SEM imaging, J. Petrol. Sci. Eng., 78, 243-257, doi:10.1016/j.petrol.2011.06.004, 2011.

Dong, M. and Chatzis, I.:, The imbibition and flow of a wetting liquid along the corners of a square capillary tube, J. Colloid Interface Sci., 172, 278-288, 1995.

Dunn K. J., Bergman D. J., and LaTorraca G. A.: Nuclear Magnetic Resonance: Petrophysical and Logging Applications, Pergamon: Elsevier Science, Amsterdam, 2002.

Fleury, M., Deflandre F., and Godefroy, S.: Validity of permeability prediction from NMR measurements, CR. Acad. Sci. Series IIC - Chemistry, 4, 869-872, doi:10.1016/S1387-1609(01)01343-3, 2001.

Ioannidis, M., Chatzis, I., Lemaire, C., and Perunarkilli, R.: Unsaturated hydraulic conductivity from nuclear magnetic resonance measurements, Water Resour. Res., 42, W07201, doi:10.1029/2006WR004955, 2006.

Jaeger, F., Bowe, S., van As, H., and Schaumann, G. E.: Evaluation of $1 \mathrm{H} \mathrm{NMR}$ relaxometry for the assessment of pore size distribution in soil samples, Eur. J. Soil Sci., 60,, 1052-1064, 2009.

Jia, P., Dong, M., and Dai, L.: Threshold pressure in arbitrary triangular tubes using RSG concept for all wetting conditions, Colloid. Surface A, 302, 88-95, 2007.

Kenyon, W.: Petrophysical principles of applications of NMR logging, Log. Anal., 38, 21-43, 1997.

Lenormand, R., Zarcone, C., and Sarr, A.: A Mechanisms of the displacement of one fluid by another in a network of capillary ducts, J. Fluid Mech., 135, 337-353, 1983.

Liaw, H.-K., Kulkarni, R., Chen, S., and Watson, A. T.: Characterization of fluid distributions in porous media by NMR techniques, AIChE J., 42, 538-546, doi:10.1002/aic.690420223, 1996.

Marquardt, D. W.: An Algorithm for the Least-Squares Estimation of Nonlinear Parameters, Siam J. Appl. Math., 11, 431-441, 1963.

Mason, G. and Morrow, N. R.: Capillary behavior of a perfectly wetting liquid in irregular triangular tubes, J. Colloid. Interf. Sci., 141, 262-274, 1991.

Mayer, R. P. and Stowe, R. A.: Mercury porosimetry-breakthrough pressure for penetration between packed spheres, J. Colloid. Interf. Sci., 20, 893-911, 1965.

Mohnke, O.: Improved forward and inverse modelling of Surface NMR relaxation signals using multi-exponential decomposition, $\mathrm{PhD}$ thesis, Technical University Berlin, Berlin, 2010.

Mohnke, O.: Jointly deriving NMR surface relaxivity and pore size distributions by NMR relaxation experiments on partially desaturated rocks, Water Resour. Res., 50, 5309-5321, 2014.

Mohnke, O. and Klitzsch, N.: Microscale Simulations of NMR Relaxation in Porous Media Considering Internal Field Gradients, Vadose Zone J., 9, 846-857, doi:10.2136/vzj2009.0161, 2010.

Or, D. and Tuller, M.: Flow in unsaturated fractured porous media: Hydraulic conductivity of rough surfaces. Water Resour. Res., 36, 1165-1177, doi:10.1029/2000WR900020, 2000.

Øren, P. E., Bakke, S., and Arntzen, O. J.: Extending predictive capabilities to network models, SPE J., 3, 324-336, 1998.

Patzek, T. W. and Silin, D. B.: Shape factor and hydraulic conductance in noncircular capillaries I. One-phase creeping flow, J. Colloid Interf. Sci., 236, 295-304, 2001. 
Princen, H. M.: Capillary phenomena in assemblies of parallel cylinders I. Capillary rise between 2 cylinders, J. Colloid. Interf. Sci., 30, 69-75, 1969a.

Princen, H. M.: Capillary phenomena in assemblies of parallel cylinders II. Capillary rise in systems with more than 2 cylinders, J. Colloid. Interf. Sci., 30, 359-371, 1969b.

Princen, H. M.: Capillary phenomena in assemblies of parallel cylinders III. Liquid columns between horizontal parallel cylinders, J. Colloid. Interf. Sci., 34, 171-184, 1970.

Ransohoff, T. C. and Radke, C. J.: Laminar flow of a wetting liquid along the corners of a predominantly gas-occupied noncircular pore, J. Colloid Interf. Sci., 121, 392-401, 1987.

Seevers, D. O.: A nuclear magnetic method for determining the permeability of sandstones, Society of Professional Well Log Analysts, Vol. 6, paper L, Housten, Texas, 1966.

Stingaciu, L. R.: Characterization of natural porous media by NMR and MRI techniques: High and low magnetic field studies for estimation of hydraulic properties, $\mathrm{PhD}$ thesis, RWTH Aachen, available at: http://darwin.bth.rwth-aachen.de/opus3/ volltexte/2010/3392/ (last access: 30 June 2014), 2010.

Stingaciu, L. R., Weihermüller, L., Haber-Pohlmeier, S., Stapf, S., Vereecken, H., and Pohlmeier, A.: Determination of pore size distribution and hydraulic properties using nuclear magnetic resonance relaxometry: A comparative study of laboratory methods, Water Resour. Res., 46, 1-11, doi:10.1029/2009WR008686, 2010.
Talabi, O., AlSayari, S., Iglauer, I., and Blunt, J.: Pore-scale simulation of NMR response, J. Petrol. Sci. Eng., 67, 168-178, 2009.

Thern, H.: Examining the fluid film model in porous media by NMR rock catalogue data, Symposium of the Society of Core Analysts, Avignon, France, paper SCA2014-051.

Tokunaga, T. K. and Wan, J.: Water film flow along fracture surfaces of porous rock, Water Resour. Res., 33, 1287-1295, doi:10.1029/97WR00473, 1997.

Toledo, P. G., Novy, R. A., Davis, H. T., and Scriven, L. E.: Hydraulic Conductivity of Porous Media at Low Water Content, Soil Sci. Soc. Am. J., 54, 673-679, doi:10.2136/sssaj1990.03615995005400030007x, 1990.

Torrey, H. C.: Bloch equations with diffusion terms, Phys. Rev., 104, 563-565, doi:10.1103/PhysRev.104.563, 1956.

Tuller, M. and Or, D.: Hydraulic conductivity of variably saturated porous media: Film and corner flow in angular pore space, Water Resour. Res., 37, 1257-1276, doi:10.1029/2000WR900328, 2001.

Tuller, M., Or, D., and Dudley, L. M.: Adsorption and capillary condensation in porous media - liquid retention and interfacial configurations in angular pores, Water Resour. Res., 35, 1949-1964, doi:10.1029/1999WR900098, 1999. 